\title{
Criminologie
}

\section{Une approche de l'étude du droit et du colonialisme : vers une perspective autohistorique amérindienne sur le changement juridique, la colonisation, les sexes et la résistance à la colonisation}

\author{
Russell Smandych et Gloria Lee
}

Volume 28, numéro 1, 1995

L'histoire de l'enfermement et des populations pénales

URI : https://id.erudit.org/iderudit/017364ar

DOI : https://doi.org/10.7202/017364ar

Aller au sommaire du numéro

Éditeur(s)

Les Presses de l'Université de Montréal

ISSN

0316-0041 (imprimé)

1492-1367 (numérique)

Découvrir la revue

Citer cet article

Smandych, R. \& Lee, G. (1995). Une approche de l'étude du droit et du colonialisme : vers une perspective autohistorique amérindienne sur le changement juridique, la colonisation, les sexes et la résistance à la colonisation. Criminologie, 28(1), 55-79. https://doi.org/10.7202/017364ar
Résumé de l'article

This paper outlines the theoretical perspective we have developed to guide the research we are undertaking on law and colonialism in the Canadian west. Although developed specifically as a tool for defining the theoretical boundaries of our own research, this theoretical perspective may be applied more generally by researchers involved in research on the history of law and social control in colonial societies. The theoretical perspective that we propose is grounded in theoretical and empirical work from a number of different fields, including the history and sociology of social control, the study of legal pluralism, and the study of the process of colonization as it has been approached in recent years by feminist scholars and First Nations historians. The purpose of this paper is to show how this new Amerindian autohistorical perspective can be used to help generate research on the interconnected issues of legal change, colonization, gender, and resistance. 
UNE APPROCHE DE L'ÉTUDE DU DROIT ET DU COLONIALISME : VERS UNE PERSPECTIVE AUTOHISTORIQUE AMÉRINDIENNE SUR LE CHANGEMENT JURIDIQUE, LA COLONISATION, LES SEXES ET LA RÉSISTANCE À LA COLONISATION ${ }^{1}$ Russel Smandych ${ }^{2}$ et Gloria Lee $^{3}$

This paper outlines the theoretical perspective we have developed to guide the research we are undertaking on law and colonialism in the Canadian west. Although developed specifically as a tool for defining the theoretical boundaries of our own research, this theoretical perspective may be applied more generally by researchers involved in research on the history of law and social control in colonial societies. The theoretical perspective that we propose is grounded in theoretical and empirical work from a number of different fields, including the history and sociology of social control, the study of legal pluralism, and the study of the process of colonization as it has been approached in recent years by feminist scholars and First Nations historians. The purpose of this paper is to show how this new Amerindian autohistorical perspective can be used to help generate research on the interconnected issues of legal change, colonization, gender, and resistance.

\section{INTRODUCTION}

Ce texte fait partie d'un travail plus vaste portant sur la transformation des mécanismes juridiques et de contrôle social dans le Canada de l'ouest, de 1670 à $1870^{4}$. L'un des objectifs de cette recherche vise à utiliser les

1. Cette étude a été rendue possible grâce à des fonds avancés par le Conseil de recherche en sciences humaines du Canada et le Solliciteur géneral du Canada, par l'entremise de dons faits au Centre de recherche en criminologie de l'Université du Manitoba. Les auteurs veulent aussi souligner l'excellente recherche foumie par James Muir, Tannis Peikoff et Ruth Swan. Le texte a été traduit de l'anglais par les Traductions Brumar.

2. Russell Smandych est professeur associế au département de sociologie de 1'Université du Manitoba, à Winnipeg, Canada. Ses travaux actuels portent sur l'histoire juridique canadienne, la sociologie du contrôle social et la criminologie comparée.

3. Gloria Lee est diplômée du programme des Études autochtones de l'Université de Saskatchewan. Elle travaille actuellement comme interprète au Centre national Héritage des Première Nations de Waneskewin, près de Saskatoon, Saskatchewan.

4. Les recherches pour ce travail s' inscrivent dans un programme réparti sur trois ans financé par le Conseil de recherche en sciences humaines du Canada. Le titre du programme est : La Transformation de l'ordre juridique et du contrôle social au Manitoba avant 1870 : une étude du développement et de l'interaction des systèmes juridiques autochtone, privé et d'ésat. Les deux principaux chercheurs sont Rick Linden et Russell Smandych, du département de sociologie de l'Université du Manitoba. 
données historiques sur la justice privée et le contrôle social contenues dans les archives de la Compagnie de la Baie d'Hudson ${ }^{5}$. En 1670, une charte royale, accordée par la monarchie anglaise, donna à la Compagnie de la Baie d'Hudson le droit exclusif de régner sur une région qui équivaut aujourd'hui approximativement à la partie ouest du Canada. La Compagnie de la Baie d'Hudson reçut le pouvoir de mettre en pratique toutes les lois et tous les règlements compatibles avec les lois d'Angleterre qu'elle jugeait nécessaires pour encadrer ses relations avec ses employés et pour maintenir l'ordre social sur le territoire de Rupertsland ${ }^{6}$. En 1821, la Compagnie obtint une licence lui permettant d'étendre son monopole et son autorité juridique sur le territoire appelé «pays indien», qui couvrait toutes les régions au-delà de Rupertsland dont les rivières se déversaient dans les océans Pacifique et Arctique (Foster, 1990) ${ }^{7}$. La Charte de 1670, augmentée des lois ultérieures, donnait en fait au Conseil des gouvemeurs de la Compagnie de la Baie d'Hudson l'autorité de gouverner un territoire représentant environ 5 pour cent de la surface terrestre du globe (Smandych et Linden, 1995).

Même si plusieurs Canadiens sont au fait de l'histoire de la Compagnie de la Baie d'Hudson et reconnaissent l'importance de son rôle, après l'établissement de rapports avec les Indiens, dans les débuts de la colonje dans l'ouest du Canada, on en sait très peu sur son rôle de législateur et d'instrument de colonisation européenne. Peu après 1670 , le Conseil des gouverneurs de la Compagnie, établi à Londres, jeta les bases juridiques d'une compagnie de commerce autogouvernée, dotée de son propre système judiciaire privé et de mécanismes de contrôle social appropriés. L'arrivée de la Compagnie de la Baie d'Hudson dans l'ouest canadien marqua aussi le début de l'intrusion de l'économie et de la culture européenne dans un territoire peuple, pendant plusieurs siècles, par des autochtones qui avaient leur propre système d'institutions sociales et culturelles, dont un droit coutumier et des méthodes traditionnelles de contrôle social et de résolution de

5. Les archives de la Compagnie de la Baie d'Hudson font maintenant partie des archives provinciales du Manitoba, a Winnipeg. Ces archives contiennent l'histoire détaillée des operations de la Compagnie de la Baie d'Hudson dans l'ouest canadien, de 1670 jusqu'à la fin du XIXe siècle.

6. "La Charte royale incorporant la Compagnie de la Baie d'Hudson, 1760 », reproduite par E. H. Oliver $(1914$, p. 135-53). Selon cette Charte, le territoire de Rupertsland comprenait toutes les terres irriguees par les rivières coulant vers la Baie d'Hudson.

7. Cette licence a été accordée en 1821, par le biais de clauses contenues dans An Act for Regulating the Fur Trade, and establishing a Criminal and Civil Jurisdiction within parts of North America (1\&2 Geo. 4. ch. 66, 1821). Cette licence a été renouvelée en 1838 pour une période de vingt et un ans. Ce n'est qu'en 1859 que la Compagnie de la Baie d'Hudson a abandonné son rôle de législateur et de justicier dans les «territoires indiens» (Foster, 1990, p. 5). 
conflits. Les employés de la Compagnie, envoyés pour établir les premiers postes de traite des fourrures le long de la côte de la Baie d'Hudson, apportèrent avec eux des ordres du Conseil de Londres dictant la façon de faire la traite et de conduire leurs relations avec les différentes tribus d'Indiens avec qui ils voulaient faire des affaires. Au cours des deux siècles suivants, jusqu'à 1870 donc, la Compagnie de la Baie d'Hudson est restée une présence dominante dans l'ouest canadien. Pendant toute cette période, ses employés ont façonné l'avenir des relations entre Européens et autochtones de l'ouest du Canada.

Dans d'autres travaux, nous avons examiné en détail le développement du système juridique de la Compagnie de la Baie d'Hudson et la façon dont il s'appliquait aux autochtones (Smandych et Lee, 1994; Smandych et Linden, 1995), et le système privé de justice créé par la Compagnie pour traiter avec ses employés délinquants (Smandych et Linden, 1994). Dans les lignes qui suivent, nous explicitons la perspective théorique de notre travail sur le droit et le colonialisme dans l'ouest canadien. Même si nous avons développé cette perspective théorique comme un outil heuristique visant à préciser les limites théoriques de notre travail, nous croyons que cette perspective historique pourrait être utile à d'autres chercheurs s'intéressant à l'histoire du droit et du contrôle social dans les sociétés coloniales. La perspective théorique que nous proposons icj s'appuie sur le travail théorique et empirique de nombreux champs, dont l'histoire et la sociologie du contrôle social, l'étude du pluralisme juridique et l'étude du processus de colonisation, tels que perçus et approchés récemment par les universitaires féministes et les historiens des Premières Nations.

Nous commençons par un aperçu des études du pluralisme juridique et du contrôle social à l'origine des questions théoriques de base dont nous discutons dans notre recherche. Suivra une discussion sur les récentes études traitant de l'impact de la colonisation européenne et de la traite des fourrures sur les Amérindiens de l'Amérique du Nord. Nous examinons en particulier les récents travaux des historiens féministes et historiens des Premières Nations, qui témoignent du besoin de développer une perspective autohistorique amérindienne plus explicite dans l'étude du droit et du colonialisme. Privilégiant une approche critique du travail de ces auteurs, nous soulignons ce que nous considérons être les éléments clés d'une perspective autohistorique amérindien ne plus explicite, et nous discutons de la façon dont on peut utiliser cette nouvelle perspective afin de susciter des recherches dans les domaines connexes du changement juridique, de la colonisation, des relations entre sexes et de la résistance à la colonisation. 


\section{ÉTUDE DU PLURALISME JURIDIQUE ET DU CONTRÔLE SOCIAL}

Au cours des récentes années, on a assisté à la parution de nombreuses études sur la sociologie du "contrôle social" (cf. Cohen et Scull, 1983; Garland et Young, 1983; Lowman, Menzies et Palys, 1987). La croissance de ce nouveau sous-champ de la criminologie, dont les sociologues sont en très grande partie responsables, est survenue en même temps que le " pluralisme juridique " est devenu l'un des sujets d'étude privilégiés des sociologues, anthropologues et spécialistes du droit et historiens (Merry, 1988, 1991). Même si, à première vue, ces deux domaines apparaissent éloignés et sans rapport, les questions théoriques et empiriques posées par les sociologues du contrôle social et les étudiants du pluralisme juridique se recoupent fréquemment (Black, 1990 ; Ellickson, 1991 ; Fitzpatrick, 1983a, 1983b ; Henry, 1983; 1987a; 1987b; Merry, 1990; O'Malley, 1987; Santos, 1987). Les lignes qui suivent donnent un aperçu des études qui ont mené aux questions théoriques de base que nous soulevons dans ce travail.

Les sociologues du contrôle social ont identifié plusieurs importantes questions théoriques et conceptuelles que la recherche empirique n'a jamais abordées. Ainsi: Comment peut-on définir le concept de «contrôle social»? Quelle est la nature précise des relations entre le droit et d'autres formes de contrôle social? Comment différentes institutions et agences de contrôle social, étatiques ou non, peuvent-elles travailler ensemble afin de créer et maintenir un ordre social ? Comment peut-on expliquer les changements dans la nature des sentences juridiques et du contrôle social? Dans notre recherche, nous poursuivons ce questionnement en nous basant sur les travaux d'auteurs comme Donald Black (1976, 1984, 1990), Stanley Cohen (1985, 1989), Michel Foucault (1977), David Garland (1985, 1990, 1991) et Stuart Henry $(1983,1987 \mathrm{a}, 1987 \mathrm{~b})$, de même que sur les premiers travaux historiques du premier de ces auteurs, portant sur le caractère changeant des principaux mécanismes de contrôle social au XIX ${ }^{e}$ siècle, dans le Haut-Canada (Smandych, 1989, 1991a, 1995).

Un autre point de départ théorique de notre recherche est la documentation croissante sur le «pluralisme juridique». Plus précisément, notre travail trouve sa source dans un intérêt à la fois pour les études traditionnelles du «pluralisme juridique classique» et pour les plus récentes et stimulantes publications du «nouveau pluralisme juridique» (Merry, 1988). Comme le montre Merry (1988), les études classées sous la rubrique "pluralisme juridique classique » se contentaient naguère pour la plupart de décrire et d'expliquer comment on superposait les nouveaux systèmes (habituellement européens) juridiques et les nouvelles lois (apportées par les colonisateurs) au droit coutumier des autochtones et leurs méthodes traditionnelles de contrôle social et de résolution de conflits. Selon Merry (1988, p. 873), «le nouveau pluralisme juridique» dont parlent des auteurs 
tels que Stuart Henry (1983), Bonaventura De Sousa Santos (1987), et Peter Fitzpatrick (1983a) «s'éloigne des recherches sur l'effet de la loi sur la société ou même de l'effet de la société sur la loi pour s'intéresser à l'interrelation complexe entre les formes officielles et non officielles de l'ordre». Certains des concepts-clés des chercheurs à l'origine du «nouveau pluralisme juridique » incluent des notions telles que «interlégalité », « ordres juridiques multiples» et « justice privée». Les défenseurs du «nouveau pluralisme juridique» utilisent l'ensemble de ces concepts pour étudier comment les nombreuses formes d'ordre juridique et de contrôle social, étatiques ou non, coexistent, interagissent et contribuent à produire $1^{\prime}$ ordre social ${ }^{8}$. Selon Santos (1987, pp. 297-299), le concept de «pluralisme juridique" constitue "le concept-clé de la vision post-moderne du droit», parce qu'il nous demande de passer de «la critique exclusive de la justice d'État existante » à la tentative de «découvrir les formes disparues ou (plus) latentes de justice qui permettent des formes plus insidieuses et plus préjudiciables d'oppression sociale et individuelle ${ }^{9}$ ".

Même s'il n'est pas nécessaire ici de discuter en détail les travaux des défenseurs du nouveau pluralisme juridique, il est important de considérer les découvertes des études portant sur l'usage du droit comme outil de colonisation. En particulier, il importe d'examiner les conclusions des études qui ont tenté d'expliquer historiquement et dans différents milieux géographiques, la manière dont les autochtones ont réagi aux tentatives d'imposition d'un système de lois étranger.

Au cours des vingt dernières années, les recherches sur le rôle du droit comme instrument du colonialisme européen se sont raffinées au plan théorique. Cette conscience théorique accrue a poussé les chercheurs à accorder plus d'attention aux nuances dans les données historiques. Les différents thèmes et les questions nouvelles que l'on trouve dans les écrits de cette période sur le droit et le colonialisme en font foi. Comme l'ont souligné Merry (1988, 1991) et d'autres (Greenberg, 1980, p. 139; Snyder et Hay, 1987), les premières recherches s'intéressaient au «rôle du droit dans la domination impériale». Plusieurs des premières études sur le droit et le colonialisme des années 1960 et 1970 « ont démontré le pouvoir de l'État à refaçonner l'ordre social, suggérant la supériorité de cette forme de droit

8. Les principaux représentants du « nouveau pluralisme juridique » sont Santos (1992) et Fitzpatrick (1992a; 1992b).

9. Récemment, le concept de «pluralisme juridique» des auteurs mentionnés ci-haut a subi les attaques de Brian Z. Tamanaha (1993), dans un article intitule « The Folly of the "Social Scientific" Concept of Legal Pluralism». En résumé, Tamanaha (1993) soutient que les aperçus fournis par ces auteurs sur le changement juridique et les formes non étatiques d'ordre normatif «ne dépendent pas du concept de pluralisme juridique et peuvent, en fait, être occultés par celui-ci»». 
sur les lois normatives » (Merry, 1988, p. 879). En plus de montrer comment un droit étatique imposé de l'extérieur devient souvent un outil de contrôle des populations coloniales efficace et puissant (cf. Kennedy, 1989), certains chercheurs ont essayé de démontrer comment les colonisateurs européens ont tenté de contrôler les autochtones en cooptant les élites locales et en cherchant à rendre plus formelles les méthodes «traditionnelles » de contrôle social et de résolution de conflits (cf. Snyder, 1981; Gordon, 1989). Plus précisément, un certain nombre de chercheurs étudiant différentes colonies ont pu montrer que «le droit coutumier, plutôt qu'être un ensemble préexistant, était un processus continu de collaboration où le pouvoir était très évidemment inégal, mais où les groupes inférieurs n'étaient pas du tout passifs ni impuissants " (Merry, 1988, p. 880).

Vers la fin des années 1970 , les tenants du pluralisme juridique classique commençaient à reconnaître plus volontiers que les peuples indigènes n'avaient pas été simplement les victimes passives d'un droit imposé de l'extérieur (Kidder, 1979). Dans les années 1980, le centre d'intérêt passa des études sur l'imposition insidieuse et coercitive du droit européen aux façons dont les peuples indigènes ont résisté à ces lois étrangères et dont ils s'en sont accommodés. Depuis le milieu des années 1980, l'étude des «formes de résistance, ouvertes ou sourdes " est devenue le principal sujet des recherches sur le droit et le colonialisme (Snyder et Hay, 1987, p. 24). Même si de récentes recherches portent encore sur la manipulation idéologique et la coercition directe des autochtones par les colonisateurs (Gordon, 1989; Kennedy, 1989), on accorde aujourd'hui plus d'attention à la « façon dont les ordres normatifs non étatiques contrent et circonscrivent la pénétration ou même s'approprient et utilisent la symbolique du droit d'État (Merry, 1988, p. 881).

On pourrait ici mentionner plusieurs recherches sur la résistance à la domination coloniale européenne : Crime as Resistance: The Colonial Situation de Peter Fitzpatrick $(1987,1989)$ et Law and Criminal Labels : The Case of the French Métis in Western Canada de Mike Brogden (1990) en sont deux exemples. On pourrait aussi poursuivre la même recherche dans la riche littérature historique qui a tenté d'évaluer l'impact du monde européen sur les populations indigènes de différents pays. Par exemple, dans les écrits des historiens universitaires sur les conséquences de l'impact européen sur les autochtones d'Australie, Attwood (1990) note elle aussi un glissement des centres d'intérêt vers le "pluralisme juridique classique »; alors que dans les études des années 1960 et 1970, on étudiait "l'impact destructeur du capitalisme et du racisme européens sur la société autochtone traditionnelle (1990, p. 121), les études plus récentes visent à donner un compte rendu plus nuancé des nombreuses « relations » et «obligations réciproques» entre Européens et autochtones, et des «stratégies 
créatrices " utilisées par les autochtones pour traiter avec les colonisateurs européens (1990, pp. 125-28) ${ }^{10}$.

Des études récentes sur le droit et le colonialisme en Afrique (Snyder et Hay, 1987), en Australie (Attwood, 1990) et ailleurs (Merry, 1991) attirent l'attention sur les différentes questions que l'on doit traiter afin de parvenir à bien comprendre les processus sociaux complexes et les circonstances historiques précises que crée l'imposition d'un nouveau type de système juridique sur un système existant de contrôle social et de résolution des conflits. Ces études illustrent aussi la variété des expériences de différents groupes autochtones un peu partout dans le monde. Les découvertes de cette recherche historique comparative et les diverses explications des auteurs justifiant leurs découvertes soulèvent de nouvelles questions pour l'étude du droit et du colonialisme dans l'ouest canadien. Par exemple, il convient de se demander à quel point les peuples indigènes de l'ouest du Canada ont essayé d'échapper et de résister à la domination des employés de la Compagnie de la Baie d'Hudson et des autres colonisateurs européens.

Un autre aspect important des écrits sur le droit et le colonialisme est l'impact des différences et des relations entre sexes sur les premiers contacts entre colonisateurs européens et peuples indigènes. Dans le sillage des conclusions de plusieurs historiennes féministes ayant étudié l'histoire des premiers rapports avec les autochtones dans l'ouest canadien (Brown, 1980; Van Kirk, 1980), Attwood (1990, p. 126) souligne, en parlant de l'cuvre d'Ann McGrath (1987), que «la différence entre les sexes a joué un rôle fondamental » dans la nature et le résultat des premières relations entre Européens et autochtones ${ }^{11} \mathrm{~d}$ 'Australie. Depuis le début des années 1980 , les universitaires féministes, qu'elles fussent sociologues, anthropologues ou historiennes, ont entrepris de brosser un portrait beaucoup plus nuancé du rôle joué par les femmes autochtones (ou indigènes) dans le processus de colonisation européenne et de l'impact de ce processus sur les relations entre sexes parmi les peuples indigènes (Leacock, 1978, 1980; Van Kirk, 1987; Anderson, 1991; Bonvillian, 1989; Bourgeault, 1988, 1989; Devens, 1992; Emberley, 1993; Foster, 1993). Dans la section suivante, nous étudierons les questions et thèmes les plus importants soulevés dans la documentation nord-américaine récente sur les femmes autochtones

10. Pour des exemples de travaux récents sur les effets du droit et du colonialisme sur les peuples maori de Nouvelle-Zélande, consulter John Pratt (1991) et Kayleen M. Hazlehurst (1993). Pour une excellente collection d'études sur l'expérience coloniale dans plusieurs pays d'Afrique, d'Asie et d'Amérique, voir Dirks (1992). Pour de bons comptes rendus de l'expérience post-colonisation des autochtones d'Amérique du Nord et du Sud, voir Bourgeault, Broad, Brown et Foster (1992).

11. Dans un autre ouvrage récent, Attwood (1992, p. 302) examine comment les auteures autochtones modernes ont contribué à « définir ou proclamer une conscience ou une identité autochtone». 
et la colonisation. Comme nous le verrons, le besoin d'adopter une perspective autohistorique amérindienne est l'une des tendances dominantes de cette littérature (cf. Sioui, 1992). Nous affirmons que l'adoption plus explicite de cette perspective pourra nous aider à mieux comprendre comment les Amérindiens eux-mêmes interprètent les événements historiques auxquels ils ont participé.

\section{LES FEMMES, LA COLONISATION ET LA RÉSISTANCE À CETTE COLONISATION : VERS UNE APPROCHE AUTOHISTORIQUE}

Au cours des dernières décennies, les socio-historiens et anthropologues historiques, appelés communément «ethnohistoriens " (cf. Axtell, 1992; Calloway, 1987; 1991; Delâge, 1993; Jaenen, 1976; Jennings, 1975; Trigger, 1976, 1985), ont essayé de comprendre les réactions des Premières Nations d'Amérique du Nord face à la colonisation européenne. Toutefois, jusqu'à maintenant, les ethnohistoriens n'ont pas réussi à définir une approche théorique précise qui servirait d'outil heuristique dans l'étude des réactions et réponses des Amérindiens au processus de colonisation européenne. Dans cet article, nous proposons un cadre théorique précis pour l'étude du droit et du colonialisme dans un contexte canadien. Le diagramme suivant (voir figure 1) illustre les éléments essentiels de la perspective autohistorique que nous proposons.

Ce diagramme s'inspire des ouvrages de plusieurs auteurs qui ont chacun souligné le besoin d'abandonner l'approche eurocentrique traditionnelle de l'histoire amérindienne. Une brève discussion de leur travail essaiera de décrire fidèlement les idées et intuitions de ces auteurs et de souligner leurs contributions à cette approche. Dès le début, toutefois, nous prévenons le lecteur que nous ne tentons nullement ici de compartimenter ni de discuter séparément chacun des concepts-clés inclus dans notre diagramme. Nous n'essayons pas non plus de découvrir un ordre causal ni une relation de cause à effet qui chercheraient à lier ces concepts entre eux. D'où l'absence de flèche ou d'autres symboles reliant les concepts-clés de notre diagramme. Cela explique aussi le cercle vide situé au centre du diagramme. Comme nous le verrons bientôt, la raison de ces décisions réside dans la logique implicite de l'approche autohistorique amérindienne. Dans la vie sociale et spirituelle des Amérindiens, toutes les formes d'interaction humaine sont nécessairement récursives et ont des effets réciproques.

La lecture du récent livre de Georges Sioui, For An Amerindian Autohistory : An Essay on the Foundations of a Social Ethic (1992), confirme la nécessité d'une approche autohistorique explicite. Sioui exige une reconnaissance du fait que la vision de l'Amérique du Nord par les colonisateurs 
européens, après l'établissement de relations avec les Amérindiens, diffère énormément de celle des Amérindiens eux-mêmes. De plus, Sioui soutient que, peu importe l'origine européenne ou autochtone de l'historien, celui-ci ne pourra jamais saisir le sens d'événements passés à moins de les voir comme les Amérindiens eux-mêmes. Selon Sioui (1992, p. 37), «le but d'une autohistoire amérindienne est d'aider l'histoire à réparer les dommages causés à l'intégrité des cultures amérindiennes».

Figure 1

\section{Essential Elements of an Amerindian Autohistorical} Perspective for the Study of Law and Colonialism



Dans son livre, Sioui explique «le système de valeurs propre aux sociétés autochtones américaines» et essaie de montrer que «les systèmes de valeurs des Amérindiens sont supérieurs aux systèmes euro-américains, et plus viables aussi ». Au centre de ces valeurs amérindiennes se trouve l'idée de «cercle sacré de la vie ", qui s'oppose "à une conception évolutionniste du monde » (1992, pp. XI, XXI). Contrairement à la tradition européenne décrivant une «prise de possession européenne » de l'Amérique du Nord, Sioui parle, au cours des décennies et siècles qui ont suivi les premiers contacts, d'une "américanisation du monde, communiquant aux autres continents l'essentiel de la pensée américaine» (1992, pp. XXII, XXIII). Dans un chapitre de son livre, The Sacred Circle of Life, Sioui souligne le rôle capital des femmes dans les sociétés "gynocentriques" amérindiennes, comme chez les Iroquoiens et les Hurons. Sioui dit précisément ceci (1992, pp. 17-18):

[...] la vénération des femmes reflète la reconnaissance par les sociêtés matricentriques d'une fratemité humaine investie dans la Terre-Mère, source de respect pour la vision personnelle dans ces sociétés. Les écrivains non autochtones perçoivent très rarement cette différence culturelle fondamentale. Ils continuent à décrire des sociétés amérindiennes, particulièrement les 
sociétés de chasseurs, gouvernées par les hommes naturellement plus imposants, alors que la réalité est toute différente [...]

Le statut élevé des femmes amérindiennes n'est pas, comme certains auteurs l'ont dit, "le résultat de leur contrôle sur l'organisation économique de la tribu». La pensée matricentrique de ces sociétés trouve sa source dans la perception aiguë qu'ont les Amérindiens du génie propre à la femme; celle-ci doit transmettre à l'homme, qu'elle éduque, les vertus humaines et sociales indispensables au maintien des relations qui sont l'essence de l'existence et de la vie.

Dans un autre ouvrage sur l'histoire amérindienne privilégiant la même perspective, Ronald Wright (1992) utilise le terme "syncrétisme» pour décrire la même idée que Sioui. Pour Wright (1992, p. 150), dans son livre Stolen Continents: The "New World"Through Indian Eyes, le "syncrétisme" représente "la croissance simultanée de croyances nouvelles et anciennes [... comme] un moyen d'encoder les valeurs de la culture conquise dans la culture dominante ». $\grave{A}$ titre de concept heuristique, le "syncrétisme" nous offre un moyen plus explicite de parler de plusieurs types de relations réciproques d'échanges qui se sont développées dans le cours des rapports entre les Européens et les Amérindiens ${ }^{12}$.

Dans le contexte de la recherche historique sur le droit et le colonialisme, la notion de "syncrétisme " peut s'appliquer tant aux relations entre les sexes qu'à l'assimilation inversée des idées de droit et de justice. Premièrement, en ce qui a trait au droit et à la justice, Merry (1991), dans sa critique des droits portant sur le droit et le colonialisme, souligne l'importance d'évaluer l'influence des peuples indigènes sur la pensée et la conduite des colonisateurs. Plus précisément, Merry (1991, p. 95) pose des questions comme : «Qui étaient les gens qui ont apporté et imposé de nouvelles conceptions du droit et quels étaient ces concepts?» "Comment leurs expériences antérieures ont-elles influencé leur façon de traiter les peuples colonisés?" "Comment leur rencontre avec le système juridique des peuples indigènes a-t-elle influencé leur conception du droit, leur identité et leur notion de suprématie culturelle et juridique? "

Toute perspective théorique désirant expliquer les liens entre droit, colonialisme et relations entre sexes dans les sociétés amérindiennes, après l'établissement des relations avec les Européens, doit tenir compte des travaux récents des ethnohistoriens féministes. L'une des questions les plus débattues au cours des récentes années est celle de l'égalitarisme des sociétés

12. Le travail des ethnohistoriens nord-américains reflète fréquemment l'usage implicite, et parfois explicite, de ce concept (cf. Axtell, 1992; Calloway, 1987; 1991; Delâge, 1993; Jaenen, 1976 ; Jennings, 1975 ; Trigger, 1976; 1985). 
amérindiennes avant l'arrivée des Européens. Dans la discussion qui suit, nous identifions et soulignons les différents points de vue qui s'affrontent sur le caractère plus ou moins églalitaire des sociétés amérindiennes d'avant la colonisation. Nous croyons importante cette réflexion parce que tous les chercheurs s'intéressant à l'histoire du droit, du colonialisme et des relations entre sexes, dans un contexte historique canadien, doivent identifier un modèle précis de société amérindienne existant avant le début de la colonisation européenne. La compréhension des relations entre sexes et du rôle respectif des femmes et des hommes dans les sociétés amérindiennes avant la colonisation est le point de départ indispensable de toute analyse de l'impact du colonialisme sur les sociétés amérindiennes ${ }^{13}$. Nous devrons aussi examiner le rôle des femmes amérindiennes dans les relations Amérindiens-Européens tout au début de la colonisation.

Beaucoup d'éléments tendent à montrer que les femmes amérindiennes ont joué un rôle capital lors des premiers contacts entre Européens et Amérindiens, dans l'est du Canada et le nord-est des États-Unis. De plus, les études récentes des universitaires féministes et historiens des Premières Nations démontrent comment les femmes amérindiennes ont influencé et modifié la conception qu'avaient les colonisateurs européens mâles des sexes et des rapports entre sexes. Comme nous le verrons bientôt, ces travaux font partie d'un mouvement qui reconnaît la nécessité d'étudier les femmes et la colonisation selon une perspective autohistorique plus explicitement amérindienne et «non eurocentrique».

Dans son récent ouvrage acclamé par tous, Olive Dickason (1992) offre un correctif à l'approche traditionnellement eurocentrique des historiens conventionnels. Même si elle n'utilise pas l'expression "perspective autohistorique" pour décrire son approche, il est clair que son livre, Canada's First Nations, tente d'interpréter l'histoire de l'Amérique du Nord à travers les yeux des Amérindiens eux-mêmes. Dans sa discussion du rôle des femmes au cours de la colonisation, Dickason $(1992$, p. 143) soutient que « les femmes ont joué un rôle capital dans la société amérindienne en particulier et commerciale en général ». Dickason (1992, p. 165) souligne que, dès les premières installations françaises à Québec, « l'idée officielle était d'encourager les hommes à épouser des femmes indigènes afin de produire une population française outre-mer ${ }^{14}$ ". Parlant du rôle des

13. Comme le note Julia Emberley (1993, p. 101) dans son récent travail déconstructionnniste sur les écrits des femmes autochtones et la théorie post-coloniale, " on ne peut assez insister, dans la critique post-coloniale, sur la nécessité d'une théorie de la subordination basée sur le sexe"

14. Dickason (1987) cite aussi les paroles de Champlain aux Hurons: «Nos jeunes hommes épouseront vos filles et nous formerons un seul peuple. 》 
Amérindiennes dans le commerce de la fourrure et la colonisation de l'ouest du Canada, Dickason (192, p. 168) note :

En résumé, les Européens devaient coopérer avec les Amérindiens afin d'établir une colonie viable et, lorsque venait le temps pour un Européen de choisir une conjointe, l'Amérindienne, ou plus tard la Métisse, avaient un avantage sur l'Européenne. Malgré les efforts [du Conseil des Gouverneurs de la Compagnie de la Baie d'Hudson] de Londres pour contrecarrer ce mouvement, les mariages interraciaux se sont multipliés parmi les Anglais lors de la colonisation de la Baie d'Hudson.

D'autres travaux récents des féministes et universitaires des Premières Nations ont ajouté à la connaissance que nous avons de l'impact de la colonisation sur les rapports entre sexes. Cependant, la plupart de ces œuvres n'adoptent pas l'approche autohistorique amérindienne que nous croyons nécessaire au réexamen de la réaction amérindienne à la colonisation européenne.

L'étude de Karen Anderson (1991) sur les femmes autochtones en Nouvelle-France, au XVII ${ }^{\mathrm{e}}$ siècle, nous en fournit un exemple. Dans son livre sur l'assujettissement des Huronnes et Montagnaises, Anderson (1991) utilise certains aspects contemporains des théories féministes et marxistes comme point de départ de son analyse de l'influence des jésuites sur les relations entre sexes, dans la période entre 1609-1649. Dans son introduction, Anderson (1991, pp. 9-8) explique :

Nous devons définir la base sur laquelle s'établissaient les différences entre les hommes et les femmes dans les sociétés autochtones et laquelle créait un rapport de pouvoir entre eux. Cette discussion s'intéressera aux champs déjà identifiés par la théorie marxiste et féministe : la production, la reproduction, la parenté et la formation de la psyché et de la vie émotive.

Anderson soutient qu'au moment des premiers contacts avec les Européens, les Amérindiennes jouissaient dans leur sociétê d'une plus grande liberté et d'une plus grande influence que les Européennes de la même époque dans la leur. Même si la vie des Amérindiennes était loin d'être facile, celles-ci n'étaient pas assujetties parce que les rôles étaient clairement définis et que les femmes et les hommes avaient leur propre sphère d'influence $^{15}$. Cela signifie simplement que les hommes et les femmes ne

15. À titre d'exemple de la division assez égale des tâches entre les sexes, Anderson souligne que, dans la société huronne, les hommes nettoyaient les champs pendant que les femmes plantaient la semence. Les hommes étaient commerçants, chasseurs et guerriers pendant que les femmes veillaient aux champs, aux récoltes, à la cueillette d'aliments sauvages et préparaient et contrôlaient la distribution des aliments et des vêtements. 
pouvaient se passer les uns des autres et que les femmes exerçaient probablement un pouvoir plus grand que les hommes ${ }^{16}$.

Anderson reconnaît que son interprétation de l'histoire huronne et montagnaise est le produit d'une lecture critique de l'ouvrage d'Eleanor Leacock $(1978,1980)$ sur le statut des femmes dans les sociétés amérindiennes. Dans cet essai très important, Leacock (1978) explique comment la structure hiérarchique considérée comme normale dans la société modeme déforme l'analyse de la structure socio-économique égalitaire et le statut des femmes dans les sociétés égalitaires. La critique de Leacock des problèmes d'interprétation crés par une perspective eurocentrique a servi subséquemment à la plupart des chercheurs de point de départ à l'examen de l'impact de la colonisation sur les relations entre sexes dans les sociétés amérindiennes. Cette critique a aussi amené plusieurs universitaires féministes à adopter une perspective autohistorique amérindienne.

L'influence du travail de Leacock (1978) se retrouve aussi dans la récente étude de Carol Devens (1992), Countering Colonization: Native American Women and the Great Lakes Missions, 1630-1900, qui essaie de combiner les thèmes de sexes et résistance. Devens soutient que les hommes autochtones étaient relativement faciles à convertir au christianisme parce que cette acceptation constituait pour eux une façon de s'adapter au nouveau contexte économique créé par l'arrivée des missionnaires jésuites et l'établissement du commerce des fourrures. D'autre part, elle soutient que les femmes autochtones résistaient beaucoup plus activement aux efforts des missionnaires qui voulaient forcer les autochtones des Grands Lacs à abandonner leurs coutumes religieuses et sociales. Elle ajoute que les femmes autochtones ont rejeté le christianisme parce qu'il compromettait leur statut dans la société et constituait une menace pour la famille. Par exemple, lorsqu'on emmenait les filles dans les écoles des missionnaires, les mères perdaient le contrôle de l'éducation des enfants. Devens (1992, p. 4) parle de ce problème:

Pendant que les hommes devenaient de plus en plus réceptifs aux nouvelles pratiques et valeurs qui leur permettraient de traiter avec les Blancs avec succès, les femmes autochtones ne pouvaient qu'y perdre autonomie et statut. Les hommes favorisaient les accommodements alors que les femmes insistaient sur la conservation des façons de faire «traditionnelles».

16. Denys Delâge, sociohistorien québécois (1993, pp. 66-69), avance un argument semblable sur le statut des femmes dans la société huronne d'avant la colonisation. En particulier, Delâge (1993, p. 66) soutient : "Vivant dans une société non coercitive, les Hurons, comme les Iroquoiens et les Algonkiens en général, étaient fiers, fortement individualistes, tolérants et libres. " De plus, il ajoute que "ces mots s'appliquent autant aux hommes qu'aux femmes ». 
Toutefois, Devens ne se contente pas d'affirmer que la structure sociale égalitaire de la société amérindienne d'avant la colonisation a commencé à se détériorer sous la pression des missionnaires chrétiens. Même si elle accepte la prémisse que les sociétés amérindiennes d'avant la colonisation étaient plus égalitaires, elle reconnaît aussi que les femmes et les hommes répondaient différemment aux événements du monde qui les entourait. Devens (1992, p. 3) souligne que son travail cherche à "voir comment les communautés autochtones percevaient les colonisateurs et comment ces perceptions ont contribué, soit à la création d'un front commun devant les colonisateurs, soit à une division menant à des factions d'accommodement et de résistance ${ }^{17}{ }^{\prime}$. En plus de brosser un tableau plus complexe de l'impact de la colonisation sur les relations entre sexes, Devens reconnaît implicitement le besoin d'adopter une perspective autohistorique amérindienne.

Selon Devens, si nous voulons comprendre le statut et le rôle des femmes dans les sociétés amérindiennes contemporaines, nous devons étudier l'influence de la colonisation sur les femmes autochtones. À mesure que les hommes abandonnaient les anciennes coutumes pour adopter la chrétienté et son éducation, les femmes s'identifiaient de plus en plus, et les hommes les identifiaient aussi, à la culture traditionnelle. D'une certaine

17. Le livre de Devens décrit des modèles de résistance et d'adaptation à la colonisation et leurs changements dans le temps. Les deux premiers modèles comprennent des réponses uniques. Dans le premier modèle, des communautés entières ont expulsé des missionnaires perçus comme des menaces au mode de vie et à la sécurité de la tribu. $\dot{A}$ d'autres moments, des conditions économiques difficiles ont forcé le groupe entier à un accommodement silencieux, et même rétif, à la chrétienté. Le troisième modèle, toutefois, of fre une réponse divisée. Lorsque les missions ou l'économie affectaient les femmes et les hommes différemment, les communautés se divisaient en factions selon les sexes qui privilégiaient des approches différentes pour faire face aux changements qui bouleversaient leur monde. Vers la fin du XIXe siècle, ce dernier modele était devenu la norme. Les hommes devenaient progressivement de plus en plus réceptifs aux pratiques et valeurs qui leur permettaient de négocier avantageusement avec les Blancs et seules les femmes risquaient de perdre leur statut et leur autonomie. Les hommes favorisaient donc l'adaptation et les femmes insistaient davantage sur le maintien des façons de faire "traditionnelles». Par consequent, les frictions entre hommes et femmes augmentèrent et devinrent chose courante parmi plusieurs communautés. Devens (1992, p. 4) soutient que ce troisième modèle clarifie le sens de la différence entre les sexes dans le processus de colonisation; cette friction entre hommes et femmes représente en fait la pomme pourrie de la colonisation. Devens affirme que si les hommes voyaient l'éducation et la conversion comme un avantage, les femmes percevaient le christianisme, les écoles et le clergé comme des menaces: l'école de la mission devint donc un champ de bataille silencieux. Les écoles de mission du XIX' siècle auraient eu un impact important sur les enfants, tirés tout à coup de leur mode de vie, et sur les mères qui perdaient soudain le contrôle de l'éducation de leurs filles (1992, pp. 108-109). 
façon, ce changement a sauvegardé le statut et les intérêts des femmes, l'isolation de leur monde les protégeant de la désintégration qui a souvent dévasté la vie des hommes. En fait, cette séparation devint si profonde qu'elle a effectivement empêché l'observation de la vie des femmes (Devens, 1992, p. 119).

Devens en vient même à reconnaître la possibilité (1992) que les dichotomies sexuelles et les conflits actuels entre sexes dans les sociétés amérindiennes découlent de la colonisation ${ }^{18}$. Les femmes sont aussi considérées comme celles qui avaient le pouvoir de se battre pour la survie de la culture traditionnelle; comme la colonisation visait d'abord les hommes, les femmes étaient tout simplement laissées de côté. Au cours de la colonisation, on intègre les hommes au système et on néglige les femmes, sauf quand les colonisateurs se rendent compte que la contribution des femmes est vitale. Voilà qui expliquerait pourquoi les colonisateurs (comme ceux de la Compagnie de la Baie d'Hudson) mentionnent rarement les femmes et pourquoi, lorsqu'on en parle, on le fait toujours en relation avec les hommes. En fait, cela expliquerait pourquoi les premières historiennes des femmes et du commerce de fourrures, comme Sylvia Van Kirk (1980) et Jennifer Brown (1980), se sont intéressées d'abord aux mariages issus de la traite des fourrures et aux familles issues de ces mariages. D'un point de vue autohistorique, la question qu'on aurait dû poser est la suivante: Pourquoi si peu de femmes autochtones ont-elles épousé des Européens? Pourquoi ont-elles résisté si farouchement, en comparaison des hommes autochtones ${ }^{19}$ ?

L'analyse de Devens (1992) souligne que les perceptions et objectifs des hommes et des femmes autochtones étaient très différents. Une telle divergence nous amène naturellement à penser que la vie traditionnelle de ces hommes et femmes étaient aussi très différente. Cette hypothèse trouve sa confirmation dans notre connaissance et notre utilisation de la perspective autohistorique amérindienne qui confirme la croyance que, même si elles jouissaient de beaucoup de liberté personnelle et de pouvoir (Sioui, 1992, pp. 15-18), les Amérindiennes étaient aussi confinées dans des rôles dictés par leur culture traditionnelle. En termes autohistoriques amérindiens, nous pourrions dire qu'une culture est le reflet et le produit de la vie spirituelle et de la vision du monde des gens qui constituent une société. Rien dans la perspective autohistorique amérindienne ne nous dit que les

18. Voir aussi Paula Gunn Allen (1986, p. 224).

19. Une documentation comparative soulevant des questions comme celles-ci voit actuellement le jour à la suite de l'étude des sexes et de la résistance à la colonisation en Californie, particulièrement durant les débuts de la colonisation espagnole en Califomie (cf. Castillo, 1994; Brady, Crome et Reese, 1984). 
hommes et femmes amérindiens d'avant la colonisation ne pouvaient avoir leur propre vision du monde basée sur leur sexe.

Pour l'ouest du Canada, on n'a fait aucun travail comparable à celui d'Anderson (1991) et Devens (1992) sur les sexes et la résistance à la colonisation. Ceux qui s'en rapprochent le plus sont les essais de Ron Bourgeault (1983, 1988, 1989) qui abordent les problèmes de race et de classe dans la société de traite des fourrures de l'ouest canadien et la domination coloniale des Indiennes. Bourgeault accepte l'interprétation de Leacock (1978) à propos de la nature des relations entre sexes avant la colonisation et utilise une perspective politico-économique marxiste de nature structuraliste pour expliquer le déclin du statut de la femme après l'arrivée de la Compagnie de la Baie d'Hudson et la croissance de la traite des fourrures. Toutefois, on a récemment contesté la validité et la valeur générale des arguments de Leacock sur la nature égalitaire des sociétés amérindiennes d'avant la colonisation.

L'un des premiers historiens à remettre en question la thèse de Leacock a été Sylvia Van Kirk. Selon cette dernière (1987, p. 1), «l'étude du rôle des femmes dans les sociétés autochtones d'avant la colonisation est vitale pour toute étude historique de la réaction des Autochtones face aux Européens ». Van Kirk (1987, p. 2) met en doute la véracité de l'affirmation de Leacock selon laquelle « les sociétés de chasseurs et glaneurs comme les Montagnais-Naskapi étaient égalitaires parce que les femmes apportaient une contribution essentielle à la survie de la bande» et parce qu'elles «avaient voix au chapitre dans le processus de prise de décision et jouissaient d'une autonomie considérable dans les domaines domestique et reproductif». Elle conteste aussi «le portrait idyllique [de Ron Bourgeault] des rôles sexuels de la société chipewyan d'avant la colonisation, présumément détruits par le commerce de la fourrure ». Van Kirk soutient «que la perspective féministe exige d'examiner soigneusement de telles affirmations et que les universitaires devraient éviter de donner un caractère absolu à des hypothèses relativistes ». Toujours selon Van Kirk (1987), une perspective féministe plus réaliste de l'histoire autochtone devrait adopter la prémisse de l'égalité probable des femmes et des hommes dans les sociétés d'avant la colonisation par rapport aux sociétés européennes « qui ont par la suite cherché à imposer leurs propres systèmes de rôles sexuels et de valeurs dans les sociétés autochtones ».

Plus récemment, Martha Foster (1993) a examiné l'impact de la colonisation sur la perception des sexes et le statut des femmes parmi les Hidatsa et les Crow. Elle (1993, p. 121) souligne que «peu d'observateurs ont noté des différences quant au rôle des femmes parmi les tribus, et ils ont essayé encore moins d'examiner les sociétés autochtones américaines à travers les yeux des femmes». Elle ajoute que l'objectif de son travail est 
d'explorer les problèmes inhérents à l'évaluation du rôle et du statut des femmes hidatsa et crow. Elle parle en particulier de «l'ignorance et de l'incompréhension fréquentes des observateurs occidentaux quant à l'importance de la tradition, du monde spirituel et du lien de parenté matrilinéaire ».

Au niveau le plus élémentaire, Foster souligne les faiblesses des travaux des archéologues et historiens qui ont liê le déclin du statut des femmes hidatsa et crow, au $\mathrm{XIX}^{\mathrm{e}}$ siècle, à certains facteurs économiques comme la traite des fourrures et l'acquisition du cheval et du fusil. Foster parle à cette occasion du «modèle des Grandes Plaines » (ou interprétation) de l'histoire amérindienne du $\mathrm{XIX}^{\mathrm{e}}$ siècle. Dans ce «contexte historique matérialiste », toujours selon Foster, le déclin du statut de la femme «s'est manifesté par une augmentation de ses tâches et de la polygamie et par une perte de contrôle sur le commerce ». Les partisans du « modèle des Grandes Plaines» soutiennent que le statut des femmes ressemblait à celui des hommes avant l'adoption du cheval et le commerce du bison, les deux phénomènes ayant contribué au déclin du statut des femmes.

L'analyse de Foster (1993, p. 123) indique que ce modèle économicodéterministe ne s'applique pas aux nations hidatsa ou crow et qu'il déforme la véritable nature du rôle et du statut des femmes dans ces sociétés. Le problème inhérent à ce modèle, selon Foster, est l'absence de preuves concrètes. Même si la plupart des premiers comptes rendus ethnographiques soutenant ce modèle étaient "colorés et dramatiques", ils «parlaient rarement des activités quotidiennes » et laissaient dans l'ombre les femmes et leurs activités.

Selon Foster (1993, p. 126), plusieurs dualismes que nous tenons pour acquis, comme "la séparation religieux/profane», et des dichotomies «comme notre vision du monde mâle/femelle ou public/privé sont des créations occidentales et ne s'appliquent pas nécessairement aux Hidatsa et aux Crow ». Selon Foster, cette interprétation des « premiers ethnologues et commerçants est à l'origine de leur incompréhension du pouvoir domestique dans les sociétés hidatsa et crow et de leur perception péjorative du travail domestique et reproducteur». Plus précisément, Foster (1993, p. 140) souligne que «c'est la pensée occidentale et mâle qui voit le travail domestique comme restrictif, non créateur et inférieur. On ne peut projeter universellement cette vision eurocentrique ni l'appliquer aux femmes hidatsa et crow ». Il est clair que Foster (1993) cherche à se placer d'un point de vue autohistorique et elle exige que nous comprenions la signification de la spiritualité et des valeurs culturelles amérindiennes.

Nous ne pouvons ici entrer en détail dans la critique de Foster du «modèle des Grandes Plaines». Toutefois, Foster (1993, p. 139) montre que, lorsque l'on examine les données historiques touchant le statut des 
femmes hidatsa et crow aux points de vue économique, politique, religieux et de liberté personnelle, il n'existe pas de preuves suffisantes pour affirmer "que leur statut se soit rapidement détérioré ». Même si Foster n'explique pas pourquoi il n'y a pas eu déclin, elle suggère que «les théories symboliques/culturelles" peuvent expliquer "l'habileté des femmes à maintenir l'égalité dans les sociétés hidatsa et crow». Ainsi, elle note (1993, pp. 142143) :

Les théories symboliques/culturelles, même si elle servent souvent à expliquer la perpétuelle domination mâle, peuvent aussi expliquer le maintien de la position des femmes, même après de profonds changements économiques et un important stress culturel. Lorsqu'une puissante idéologie soutient l'autonomie personnelle des femmes ainsi que leurs positions économique et religieuse, comme dans les sociétés matrilinéaires telles que les Crow, un modèle basé seulement sur des facteurs économiques se révèle incomplet. Le statut est le produit de plusieurs facteurs. Prétendre qu'une théorie, qu'elle soit économique, biologique ou psychologique, répond à toutes les questions touchant le statut ne tient pas compte de cette complexité.

Les chercheurs précédents, à l'exception de Foster, ont tous négligé cet élément spirituel. Si l'on veut trouver des raisons à l'égalité ou à l'inégalité des femmes et des hommes que l'on observe dans les sociétés autochtones, on doit comprendre l'importance de leurs croyances spirituelles. L'élément de base de toute la culture et de la structure sociale amérindienne est la spiritualité des individus et la spiritualité collective de la société en général. Dans la vie amérindienne, la spiritualité englobe tout et fournit le cadre de référence. De façon fondamentale, elle dicte les relations interpersonnelles et le respect. En même temps, les croyances spirituelles fondent les différences trouvées dans la culture et les relations amérindiennes.

On pourrait prétendre que la culture et les relations individuelles s'équilibraient de façon à préserver l'harmonie sociale. Toutefois, on ne peut pas dire que toutes les choses étaient égales. Il est important de comprendre que «l'équilibre des forces" présent dans les sociétés amérindiennes reposait sur un autre système de valeurs que celui qui domine la société occidentale. On peut aussi affirmer que les femmes jouissaient d'un pouvoir important reconnu et accepté par tous.

Avec la spiritualité comme base de connaissance, la perspective historique nous amène à poser l'hypothèse que les relations hommes/femmes fonctionnaient à l'intérieur d'un équilibre des pouvoirs. Dans les sociétés d'avant la colonisation, l'équilibre des pouvoirs entre femmes et hommes était en constant changement. Ce n'est qu'au contact de la culture patriarcale des colonisateurs européens que les femmes furent réduites à un rôle qu'elle n'avait jamais connu auparavant. Cet assujettissement apparut aux 
autochtones mâles comme un moyen de survie (cf. Anderson, 1991; Devens, 1992) car les colonisateurs européens mâles le pratiquaient. Cette compréhension des explications possibles de la nature des relations entre sexes dans les sociêtés amérindiennes d'avant la colonisation constitue la toile de fond nécessaire à toute étude de la place des femmes, de la colonisation et de la résistance à cette colonisation.

Toute étude du droit et du colonialisme au Canada doit considérer des constantes comme les valeurs culturelles et les croyances des sociétés amérindiennes; là réside la force qui a soutenu les Amérindiens pendant les guerres, famines, maladies et bouleversements spirituels. Selon Sioui (1992), tous les systèmes de croyances amérindiens reposent sur ce postulat fondamental que vie spirituelle, humains et animaux font partie de ce "cercle sacré » et que l'on doit reconnaître et respecter toutes ces relations entre mondes physique et spirituel. Si nous acceptons ce principe de Sioui, nous devons conclure que les sociétés amérindiennes d'avant la colonisation étaient essentiellement, jusqu'à un certain point, plus égalitaires.

\section{CONCLUSIONS ET PISTES POUR RECHERCHES FUTURES}

Nous avons tenté ailleurs (Smandych et Lee, 1994; Smandych et Linden, 1994, 1995) de décrire les circonstances historiques qui ont conduit la Compagnie de la Baie d'Hudson à créer un système de justice privé et à le faire prévaloir sur le système de résolution des conflits des peuples indigènes de l'ouest du Canada. Dans cette recherche, nous nous sommes penchés sur des questions et des thèmes précis soulevés dans les récents travaux des sociologues du contrôle social et des étudiants du pluralisme juridique. De plus, nous utilisons la perspective autohistorique présentée dans ce travail comme point de départ de notre interprétation de la perception par les autochtones de l'imposition du droit par la Compagnie de la Baie d'Hudson. Cette perspective reconnaît que, lorsque nous étudions des questions historiques comme la nature des méthodes traditionnelles de résolution de conflits et de contrôle social chez les autochtones, ou les différents rôles des hommes et des femmes dans les sociétés amérindiennes avant l'arrivée des Européens, nous devons nous contenter de la vision déformée des documents laissés par les colonisateurs européens. Dans notre travail, nous utilisons cette perspective afin de montrer l'inadéquation des approches théoriques et méthodologiques des historiens conventionnels, et nous proposons les correctifs nécessaires pour pallier cette insuffisance.

Nous en sommes encore aux premiers pas de notre recherche portant sur l'impact de la Compagnie de la Baie d'Hudson et de son système juridique privé sur les autochtones vivant dans ce qu'on appelle maintenant l'Ouest du Canada. L'une de nos principales découvertes est la soumission des autochtones au système privé imposé par la Compagnie dès son arrivée 
dans l'ouest canadien (Smandych et Lee, 1994; Smandych et Lee, 1995). Toutefois, nous avons aussi découvert que les autochtones de l'ouest canadien n'ont pas été les victimes passives d'un procédé de colonisation par le droit; ils ont plutôt résisté activement à l'imposition de ce système par les colonisateurs européens. Nous poursuivons actuellement notre recherche dans les archives afin de répondre aux importantes questions thériques soulevées récemment par les sociologues du contrôle social et les tenants du pluralisme juridique, de même que par les historiens féministes et amérindiens.

Notre travail actuel privilégie la perspective autohistorique amérindienne dans notre étude de l'impact de la colonisation sur les femmes autochtones et de leur implication comme agentes de syncrétisme et de résistance. De plus, nous analysons d'un point de vue déconstructionniste les documents historiques où l'on parle du rôle et du statut des femmes dans les sociétés amérindiennes immédiatement après leur contact avec les Européens (Emberley, 1993). Nous examinons aussi les questions posées par Merry (1991) touchant l'impact de la colonisation sur les colonisateurs européens eux-mêmes. Les historiens canadiens de la traite des fourrures ont déjà montré que, pendant des années, les employés de la Compagnie de la Baie d'Hudson ont survécu grâce aux connaissances et aux ressources des peuples indigènes (Ray et Freeman, 1978; Russell, 1991; Thistle, 1986). Ceux-ci ont dû aussi provoquer une révision des idées de droit et de justice chez les Européens. Il reste beaucoup à apprendre sur les gens qui « ont apporté et imposé de nouvelles conceptions du droit» et sur les effets «que leurs expériences antérieures ont eu sur leur façon de traiter les peuples colonisés" (Merry, 1991, p. 55). En bout de ligne, nous espérons que l'attention que nous portons à ces questions permettra d'en connaître davantage sur les questions connexes du changement juridique, de la colonisation, des relations entre les sexes et de la résistance à la colonisation. 


\section{BIBLIOGRAPHIE}

ALLEN, Paula Gunn (1986), The Sacred Hoop: Recovering the Feminine in American Indian Traditions, Boston, Beacon Press.

ANDERSON, Karen (1991), Chain Her by One Foot:.The Subjugation of Women in Seventeenth-Century New France, Londres, Routledge.

ATTWOOD, Bain (1990), «Aborigines and Academic Historians: Some Recent Encounters », Australian Historical Studies, vol. 24, pp. 123-135.

ATTWOOD, Bain (1992), «Portrait of an Aboriginal as an Artist : Sally Morgan and the Construction of Aboriginality", Australian Historical Studies, vol. 25 , pp. 302-318.

AXTELL, James (1992), Beyond 1492 : Encounters in Colonial North America, New York, Oxford University Press.

BLACK, Donald (1976), The Behavior of Law, New York, Academic Press.

BLACK, Donald (éd.) (1984), Toward a General Theory of Social Control, New York, Academic Press, 2 vol.

BLACK, Donald (1990), «Elementary Forms of Conflict Management ", New Direc. tions in the Study of Justice, Law, and Social Control, School of Justice Studies, Arizona State University, New York, Plenum Press.

BONVILLIAN, Nancy (1989), "Gender Relations in Native North America ", American Indian Culture and Research Journal, vol. 13, pp. 1-28.

BOURGEAUlT, Ron (1983), "The Indian, the Metis and the Fur Trade: Class, Sexism and Racism in the Transition from "Communism" to Capitalism", Studies in Political Economy, vol. 12, pp. 45-79.

BOURGEAULT, Ron (1988), "Race and Class Under Mercantilism: Indigenous People in Nineteenth-Century Canada ", in S. Bolaria et P. Li (éd.) Racial Oppression in Canada, $2^{\mathrm{e}}$ édition. Toronto, Garamond Press.

BOURGEAULT, Ron (1989), «Race, Class, and Gender: Colonial Domination of Indian Women ", in s. Vorst et al. (éd.) Race, Class, Gender : Bonds and Barriers, Halifax, Garamond.

BOURGEAULT, Ron, Dave BROAD, Lome BROWN, et Lori FOSTER (éd.) (1992), 1492-1992 - Five Centuries of Imperialism and Resistance, Toronto, Fernwood.

BRADY, Victoria, Sarah CROME, et Lynn REESE (1984), « Resist! Survival Tactics of Indian Women ", California History (Spring), pp. 141-51.

BROGDEN, Mike (1990), «Law and Criminal Labels : The Case of the French Métis in Western Canada ", Journal of Human Justice, vol. 1, pp. 13-32.

BROWN, Jennifer (1980), Strangers in Blood: Fur Trade Company Families in the Indian Country, Vancouver, University of British Columbia Press.

CASTILLO, Edward D. (1994), «Gender Status Decline, Resistance, and Accommodation among Female Neophytes in the Missions of Califomia : A San Gabriel Case Study ", American Indian Culture and Research Journal, vol. 18, pp. 67-93.

CALLOWAY, Colin G. (1987), Crown and Calumet : British-Indian Reiations, 17831815, Norman, University of Oklahoma Press.

CALloWAY, Colin G. (éd.) (1991), Dawnland Encounters : Indians and Europeans in Northern New England, Hanover, University Press of New England. 
COHEN, Stanley (1985), Visions of Social Control : Crime, Punishment and Classification, Cambridge, Polity Press.

COHEN, Stanley (1989), "The Critical Discourse on "Social Control" : Notes on the Concept as a Hammer", International Journal of the Sociology of Law, vol. 17, pp. 339-363.

COHEN, Stanley et Andrew SCULL (éd.) (1983), Social Control and the State, New York, St. Martin's Press.

DELÂGE, Denys (1993), Bitter Feast : Amerindians and Europeans in Northeastern North America, 1600.64, traduit du français par Jane Brierley, Vancouver, University of British Columbia Press. Le Pays renversé, Les éditions du Boréal, 1985.

DEVENS, Carol (1992), Countering Colonization : Native American Women and Great Lakes Missions, 1630-1900, Berkeley, University of California Press.

DICKASON, Olive (1992a), Canada's First Nations, Toronto, McClelland and Stewart.

DIRKS, Nickolas B. (éd.) (1992), Colonialism and Culture, Ann Arbor, University of Michigan Press.

ELLICKSON, Robert C. (1991), Order Without Law : How Neighbours Settle Disputes, Cambridge, Harvard University Press.

EMBERLEY, Julia V. (1993), Thresholds of Difference : Feminist Critique, Native Women's Writings, Postcolonial Theory, Toronto, University of Toronto Press.

FITZPATRICK, Peter (1983a), «Law and Societies», Osgoode Hall Law Journal, vol. 22, pp. 115-138.

FITZPATRICK, Peter (1983b), «Law, Plurality and Underdevelopment ", in D. Sugerman (éd.) Legality, Ideology and the State, Toronto, Academic Press.

FITZPATRICK, Peter (1987), "Transfornations of Law and Labour in Papua New Guinea ", in F. Snyder et D. Hay (ed.) Labour, Law, and Crime : A Historical Perspective, Londres, Tavistock.

FITZPATRICK, Peter (1989), "Crime as Resistance: The Colonial Situation», The Howard Journal of Criminal Justice, vol. 28, pp. 272-281.

FITZPATRICK, Peter (1992a), The Mythology of Modern Law, Londres, Routledge.

FITZPATRICK, Peter (1992b), "The Impossibility of Popular Justice», Social \& Legal Studies, vol. 1, pp. 199-216.

FOSTER, Hamar (1990), «Long-Distance Justice : The Criminal Jurisdiction of Canadian Courts West of the Canadas, 1763-1859 ", The American Journal of Legal History, vol. 34, pp. 1-48.

FOSTER, Martha Harroun (1993), «Of Baggage and Bondage: Gender and Status Among Hidatsa and Crow Women ${ }_{*}$ American Indian Culture and Research Journal, vol. 17, pp. 121-152.

FOUCAULT, Michel (1977), Discipline and Punish: The Birth of the Prison, New York, Vintage Books.

GARLAND, David (1985), Punishment and Welfare: A History of Penal Strategies, Aldershot, Gower.

GARLAND, David (1990), Punishment and Modern Society : A Study in Social Theory, Chicago, University of Chicago Press.

GARLAND, David (1991), «Punishment and Culture: The Symbolic Dimension of Criminal Justice », Studies in Law, Politics, and Society, vol. 11, pp. 191-222. 
GARLAND, David et Peter YOUNG (éd.) (1983), The Power to Punish : Contemporary Penality and Social Analysis, Londres, Heinemann.

GORDON, Robert (1989), «The White Man's Burden : Ersatz Customary Law and internal pacification in South Africa", Journal of Historical Sociology, vol. 2, pp. 41-65.

GREENBERG, David F. (1980), «Law and Development in Light of Dependency Theory $"$, Research in Law and Sociology, vol. 3, pp. 129-159.

HAZLEHURST, Kayleen M. (1993), Political Expression and Ethnicity : Statecraft and Mobilization in the Maori World, Westport, CT., Greenwood Press.

HENRY, Stuart (1983), Private Justice : Towards Integrated Theorizing in the Sociology of Law, Londres, Routledge \& Kegan Paul.

HENRY, Stuart (1987a), a Disciplinary Pluralism : Four Models of Private Justice in the Workplace", Sociological Review, vol. 35, pp. 279-319.

HENRY, Stuart (1987b), "The Construction and Deconstruction of Social Control : Thoughts on the Discursive Production of State Law and Private Justice», in J. Lowman, R. Menzies et T. Palys (éd.) Transcarceration : Essays in the Sociology of Social Control, Aldershot, Gower.

HILL, Jonathan D. «Overview. Contemporary Issues Forum. Contested Pasts and the Practice of Anthropology ", American Anthropologist, vol. 94, pp. 809-815.

JAENEN, Comelius (1976), Friend and Foe: Aspects of French-Amerindian Cultural Contact in the Sixteenth and Seventeeth Centuries, Toronto, McClelland and Stewart.

JENNINGS, Francis (1975), The Invasion of America : Indians, Colonialism, and the Cant of Conquest, New York, Norton and Co.

KENNEDY, Mark (1989), "Law and Capitalist Development : The Colonization of Sub-Saharan Africa», in T. Caputo, M. Kennedy, C. Reasons et A. Brannigan (6d.) Law and Society : A Critical Perspective, Toronto, Harcourt Brace Jovanovich.

KIDDER, Robert L. (1979), "Toward an Integrated Theory of Imposed Law", in S. Burman et B. Harrell-Bond (éd.) The Imposition of Law, New York, Academic Press.

LEACOCK, Eleanor (1978), « Women's Status in Egalitarian Society : Implications for Social Evolution », Current Anthropology, vol. 19, pp. 247-275.

LEACOCK, Eleanor (1980), « Montagnais Women and the Jesuit Program for Colonization », in M. Etienne et E. Leacock (éd.) Women and Colonization : Anthropological Perspectives, New York, Praeger.

MCGRATH, Ann (1987), «Born in the Cattle»: Aborigines in Cattle Country, Sydney, Allen \& Unwin.

MERRY, Sally (1988), «Legal Pluralism», Law and Society Review, vol. 22, pp. 869896.

MERRY, Sally (1990), Getting Justice and Getting Even : Legal Consciousness among Working Class Americans, Chicago, University of Chicago Press.

MERRY, Sally (1991), "Law and Colonialism», Law and Society Review, vol. 25, Pp. 889-922.

OLIVER, E. H. (1914), The Canadian North-West : Its Early Development and Legislative Records, Ottawa, Government Printing Bureau, vol. 1. 
O'MALLEY, Pat (1987), «Regulating contradictions: The Australian Press Council and the Dispersal of Social control ", Law and Society Review, vol. 21, pp. 82108.

PRATT, John (1991), «Citizenship, Colonisation and Criminal Justice », International Journal of the Sociology of Law, vol. 19, pp. 293-319.

RAY, Arthur et Donald FREEMAN (1978), Give us Good Measure : An Economic Analysis of Relations Between the Indians and the Hudson's Bay Company Before 1763, Toronto, University of Toronto Press.

RUSSELL, Dale R. (1991), Eighteenth-Century Western Cree and Their Neighbours, Hull, Canadian Museum of Civilization.

SANTOS, Bonaventura De Sousa (1987), "Law : A Map of Misreading. Toward a Postmodem Conception of Law ", Journal of Law and Society, vol. 14, pp. 279302.

SANTOS, Bonaventura De Sousa (1992), "State, Law and Community in the World System : An Introduction », Social \& Legal Studies, vol. 1, pp. 131-142.

SMANDYCH, Russell C. (1989), «The Upper Canadian Experience with Pre-Segregative Control », thèse de doctorat, University of Toronto.

SMANDYCH, Russell C. (1991a), "Tory Patemalism and the Politics of Penal Reform in Upper Canada, 1830-1834: A "Neo-Revisionist" Account of the Kingston Penitentiary", Criminal Justice History: An International Annual, vol. 12, pp. 57-83.

SMANDYCH, Russell C. (1991b), « Rethinking "the Master Principle of Administering Relief" in Upper Canada: A Response to Allan lrving", Canadian Review of Social Policy, $\mathrm{n}^{\circ} 27, \mathrm{pp} .81-86$.

SMANDYCH, Russell C. (1995), «William Osgoode, John Graves Simcoe, and the Exclusion of the English Poor Law from Upper Canada », in L. Knafla et $S$. Binnie (éd.) Law, State, and Society : Essays in Modern Legal History, Toronto, University of Toronto Press.

SMANDYCH, Russell et Gloria LEE (1994), «Resisting Company Law : Aboriginal Peoples and the Transformation of Legal Ordering and Social Control in the Canadian West to 1850 », communication présentée lors du congrès annuel de la Western Association of Sociology and Anthropology, Saskatoon, 3-5 mars.

SMANDYCH, Russell et Rick LINDEN (1994), «Company Discipline in the Hudson's Bay Company $1670-1770 \%$, communication présentée lors du congrès annuel de la Canadian Law and Society Association, Calgary, 13-15 juin.

SMANDYCH, Russell et Rick LINDEN (1995), «Co-Existing Forms of Native and Private Justice: An Historical Study of the Canadian West", in Kayleen M. Hazlehurst (éd.) Legal Pluralism and the Colonial Legacy: Indigenous Experiences of Justice in Canada, Australia, and New Zealand. Cambridge, Cambridge University Press, sous presse.

SNYDER, Francis G. (1981), "Colonialism and Legal Form : The Creation of "Customary Law" in Senegal », Journal of Legal Pluralism, vol. 1, pp. 49-92.

SNYDER, Francis G. et Douglas HAY (1987), «Comparisons in the Social History of Law : Labour and Crime », in F. Snyder et D. Hay (éd.) Labour, Law and Crime, Londres, Tavistock.

SIOUI, Georges E. (1992), For an Amerindian Autohistory : An Essay on the Foundations of a Social Ethic, Montreal, McGill-Queen's. 
TAMANAHA, Brian Z. (1993), "The Folly of the "Social Scientific" Concept of Legal Pluralism ", Journal of Law and Society, vol. 20, pp. 192-216.

THISTLE, Paul (1986), Indian-European Trade Relations in the Lower Saskatchewan River Region to 1840, Winnipeg, University of Manitoba Press.

TRIGGER, Bruce (1976), The Children of Aataentsic : A History of the Huron People to 1660, Kingston et Montreal, McGill-Queen's University Press.

TRIGGER, Bruce (1985), Natives and Newcomers: Canada's «Heroic Age » Reconsidered. Kingston et Montréal, McGill-Queen's University Press.

VAN KIRK, Sylvia (1980), "Many Tender Ties": Women in Fur Trade Society in Western Canada 1670-1870, Winnipeg, Watson \& Dwyer.

VAN KIRK, Sylvia (1987), "Toward a Feminist Perspective in Native History", Toronto, Centre for Women's Studies in Education, Occasional Paper, $\mathrm{n}^{\circ} 14$.

WRIGHT, Ronald (1992), Stolen Continents: The *New World" Through Indian Eyes, Toronto, Penguin. 